\title{
Estimation pratique des erreurs commises dans la mesure du vent en altitude avec un radar à poursuite automatique en utilisant la méthode des sondages rapproches dans le temps*
}

\author{
Par G. D. Barbé \\ Météorologie Nationale, Paris, France \\ (Manuscript received 3 May 1957)
}

\section{Methodologie}

Quand on mesure une longueur constante avec un mètre imparfait-dont la longueur est affectée de variations fortuites dans le temps-les résultats de mesures, s'il s'agit de "plusieurs" mesures permettent d'apprécier les variations de longueur autrement dit les "erreurs" affectant le mètre.

Dans ce cas c'est la longueur à mesurer qui joue le rôle d'unité de référence et le mètre celui de grandeur inconnue.

Si la grandeur à mesurer avec le mètre erroné est non pas constante mais affectée d'une variation progressive dans le temps (systématique) il est possible de mettre là encore en évidence une limite supérieure approchée de l'erreur fortuite affectant le mètre étalon: à la condition que ces dernières erreurs ne masquent pas la variation systématique de la grandeur à mesurer.

Ce principe a pu être-dans une certaine mesure, mais suffisamment pour y trouver un intérêt pratique-appliqué à la mesure du vent en altitude à l'aide d'un radar à poursuite automatique dont la précision est définie et relativement grande.

De nombreux sondages-plus de 700 entre le 15 Octobre 1956 et le 15 Mai 1957 ont été effectués à Trappes, près de Paris, avec un radar Français à poursuite automatique ${ }^{(1)}$ dont la précision est de:

-100 à $200 \mathrm{~m} /$ minute (6 à $12 \mathrm{~km} / \mathrm{h}$ ) suivant l'altitude dans la mesure de la grandeur du vecteur vent.

—et généralement supérieure à $10^{\circ}$ dans la mesure de la direction du vent pour des vents de force supérieure à $300 / \mathrm{m}$ minute.

Ceci correspond à une précision généralement supérieure à:

$-50 \mathrm{~m}$ dans la mesure de la distance oblique radar-ballon

- et à $2 / 1000$ de radian dans la mesure des angles de site et d'azimut.

L'utilisation que nous avons faite de la méthode des sondages de vent rapprochés dans le temps:

-l'intervalle de temps entre 2 sondages successifs était généralement compris entre 1h. et 1 h. $1 / 2^{(2)}$

* Communication presentée à la Session Commune CIMO-Ae de L’Organisation Météorologique Mondiale, Paris, Juin 1957.

(1) construit par la Compagnie FrançaiseTHOMSON-HOUSTON, longueur d'onde $10 \mathrm{~cm}$.

(2) Toutefois des sondages espacés de 15 minutes ont été exécutés pour l'étude des courants jets à l'intérieur du tube de jet dans une couche de 5 à $6 \mathrm{~km}$ d’épaisseur axée sur le niveau du maximum de vent.

Nous avons, de plus, pu mettre au point un procédé permettant de suivre au radar successivement 3 ballons lancés à 5 minutes d'intervalle; chaque ballon donnant lieu à une mesure du vent pendant 5 minutes successives. Trois couches de l'atmosphère chacune d'une épaisseur de l'ordre de $2 \mathrm{~km}$. environ, situées à des niveaux séparés par une différence d'altitude de $6 \mathrm{~km}$. environ, ont ainsi pu être explorées à l'aide de mesures effectuées à 5 minutes d' intervalle.

Ce procédé peut être facilement généralisé: nous avons l'intention de l'appliquer à l'étude particulière suivante:

mesure à l'intérieur d'une couche de $2 \mathrm{~km}$. d'épaisseur environ axée sur le niveau du maximum de vent d'un courant jet, pendant une durée de 2 heures environ, voisine du moment où le vent passe dans le temps par un maximum à la verticale d'un point, de la direction du vecteur vent "ainsi que de la grandeur 「du "vent transversal” (composante du vent perpendiculaire au rayon vecteur radar-ballon). Cette mesure sera relative à des couches de 200 mètres environ d’épaisseur, à raison d'une mesure toutes les 10 minutes. 
a mis en évidence une proprièté-qui paraît fondamentale-de la circulation atmosphérique en altitude (au-dessus des 2 ou 3 premiers kilomètres à partir du niveau du sol).

Cette propriété concerne la "stabilité" de la circulation atmosphérique:

Le degré de stabilité mis en évidence par la superposition des courbes correspondant à des sondages successifs est élevé: il se manifeste avec un degré de détail qui surprend.

En d'autres termes la "turbulence atmosphérique" n'intervient dans la circulation atmosphérique en altitude au-dessus de 2 à $3 \mathrm{~km}$ généralement ${ }^{(1)}$ qu'à une échelle de temps et d'espace (en altitude et dans un plan horizontal) inférieure à l'échelle des mesures actuelles: couches de 300 à 500 mètres d'épaisseur, vitesse du vent appréciée à $6 \mathrm{~km} /$ heure près, intervalle de temps entre 2 sondages de l'ordre d'une heure.

C'est donc grâce à la nature intrinsèque de la circulation atmosphérique en altitude que peut être appréciée la précision d'un instrument de mesure du vent en altitude à partir du moment où cette précision dépasse un certain seuil:

- pratiquement pour un radar:(2)

$-50 \mathrm{~m}$ dans la mesure de la distance oblique

- l à 2/1000 de radian ( 0,05 à 0,1 degré) dans la mesure des angles de site et d'azimut.

(1) Il y a quelques exceptions, se reporter à l'Etude sur: “Mesure précise du vent en altitude à l'aide de sondages rapprochés dans le temps" mars 1956-p. 9. B. 2., p. 12. II.4.2., p.13.

(2) Il s'agit ici de la précision "totale" tenant compte à la fois des erreurs de la partie électrique du radar, de celles dues au jeu des mécanismes, au procédé de lecture et enfin à l'agitation du réflecteur.
Exemples prèsentés-Détermination pratique de la précision du radar francais:

Thomson-Houston "Cotal" modifié à poursuite automatique de longueur d'onde $10 \mathrm{~cm}$

A-Expressions des éléments du vecteur vent (Fig. 1)

En désignant par:

$\mathrm{R}, \mathrm{S}, \alpha$ les données primaires du radar fixant la position du ballon dans l'espace à un instant donné.

$\mathrm{R}$ distance oblique $O B_{1}$-radar-ballon

$\mathrm{S}$ angle de site $\left(\overrightarrow{O B}_{1}, \overrightarrow{O b}_{1}\right)$

$\alpha$ angle d'azimut ou de gisement $\left(\overrightarrow{O x}, \overrightarrow{O b}_{1}\right)$

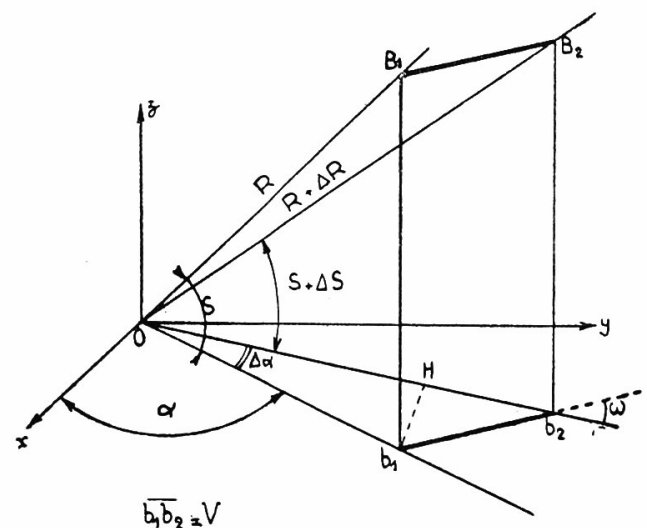

$\bar{a}_{1} b_{2}=V$

$\overline{b_{1} H}=V_{t} \# \cos S . \Delta \alpha$

$\overrightarrow{b_{2}} \mathrm{H}=V_{r}=(R+\Delta R) \cdot \cos (S \div \Delta S)-R \cos S$ $=\Delta R \cos (S+\Delta S)-Z \Delta S$

$\lg \omega=\frac{V_{t}}{V_{r}}=\frac{R \cos S \cdot \Delta \alpha}{\Delta R \cos (S+\Delta S)-Z \Delta S}$

$\operatorname{tg} \omega \#\left(\frac{R}{\Delta R}\right) \dot{\Delta} \alpha$

Fig. 1

par $D R, D S, D \alpha$ la variation de ces quantités pendant l'intervalle de temps entre 2 lectures (1 minute en général).

En désignant de plus:

- par $V r=l e$ vent "radial": composante du vent projetée sur la direction horizontale $O b_{1}$ vers laquelle regarde le radar;

- par $V_{t}=$ le vent "transversal" ou tangentiel: composante du vent perpendiculaire à la précédente; $\mathrm{du}$

hor

I1

van

ou

puis

$\mathrm{du}$

pass

et 1

$\mathrm{du}$

de

\section{B.}

avec

$2 \mathrm{cc}$

$\mathrm{L}$

son

sim

deg

$\mathrm{L}$ 
- par o l'angle que fait la direction du vent avec le rayon radar-projection horizontale du ballon $O b_{1}$.

Il est facile d'établir les expressions suivantes:

$$
V r=D R \cdot \operatorname{Cos}(S+D S)-R \cdot \sin . S . D S
$$
ou encore:

$$
V r=D R \cdot \cos (S+D S)-z D S
$$

puisque $z=R$. Sin. $S, z$ désignant la hauteur du ballon au-dessus du plan horizontal passant par le radar.

$$
V t=R \cdot \cos S . D \alpha
$$

$$
t_{g \omega}=\frac{R \cdot \cos \cdot S D \alpha}{D R \cdot \cos (S+D S)-z D S}
$$

et la formule approchée valable quand:

$$
\frac{\frac{z D S}{D R \cdot \cos (S+D S)} \# 0}{\operatorname{tg} \omega \# \frac{R}{D R} \cdot D \alpha}
$$

Le module $|\vec{V}|$ du vent et la direction $D$ du vent s'expriment facilement en fonction de $V_{t}, V_{r}$. et $\omega$

$$
\begin{gathered}
|\vec{V}|=\sqrt{V_{r^{2}+V_{t}^{2}}}(1) \\
\text { et } \quad D=\alpha \pm \omega \pm \pi
\end{gathered}
$$

$\mathrm{B}-$ Représentation de la variation $d u$ vent avec l'altitude par 3 courbes équivalentes aux 2 courbes $V(z)$ et $D(z)$

Les éléments classiques du vecteur vent sont:

-le module $|\vec{V}|$ que nous écrirons plus simplement $V$ par la suite

- la direction $D$ repérée en rose de 360 degrés.

La nécéssité absolue, où nous nous trouvi-

(1) Ce qui peut encore s'écrire, lorsque $\frac{V_{t}}{V_{r}} \leq 1$ avec une approximation correspondant à une erreur relative maximum de $6 \%$ sur $V$ atteinte lorsque $\frac{V_{t}}{V_{r}}=1:$

$$
|\vec{V}|=V r\left[1+\frac{1}{2}\left(-\frac{V_{t}}{V r}\right)^{2}\right]
$$

ons $^{(2)}$ de suivre en cours de sondage, les variations du vent mesuré de minute en minute, par comparaison avec les sondages antérieurs nous a conduits à fonder cette comparaison:

-non pas sur les éléments classiques du vecteur vent qu'il n'aurait pas été possible de calculer à temps,

- mais sur les éléments suivants ${ }^{(3)}$ qui suffisent à déterminer le vent

a) -la variation-en une minute-DR de la distance oblique $R$ radar ballon;

b) -le vent transversal $V_{t}$

c) -le terme "correctif" $-z D S$ homogène à une vitesse intervenant dans l'expression du vent radial:

Il est facile de voir que si pour deux sondages successifs que nous dénommerons, par la suite, pour la facilité de l'exposé rouge et bleu, les courbes bleue et rouge représentant la variation en fonction de l'altitude respectivement de $D R, V_{t}$ et $-z D S$ coincident,

Il en sera de même des courbes bleue et rouge donnant la variation de $V$ et de $D$.

C'est ce que l'expérience nous a permis de vérifier généralement à posteriori.

En conclusion, nous pouvons avancer: que le degré de coincidence entre elles pour deux ou plusieurs sondages successifs, de chacune des courbes blene et rouge.

$$
D R=f(z) \quad-z D s=f(z) \quad \text { et } \quad V_{t}=f(z)
$$

mesure le degré de coincidence des courbes $V=f(z)$ et $D=f(z)$ et permet donc l'appréciation pratique de la précision des mesures.

\section{Résultats expérimentaux-}

L'expérience de plus de 6 mois des sondages rapprochés dans le temps pour un total

(2) par suite de l'exécution de nombreux sondages successifs rapprochés dans le temps, principe fondamental de la méthode de mesure utilisée.

(3) apportant une "quantité d'information" beaucoup plus grande-essentiellement par la prise en considération du vent transversal $V_{t}$ : on reviendra plus loin sur ce point. 
de sondages dépassant $700^{(1)}$ nous a amenés à faire la constatation-de grande importance pratique-sur laquelle nous ne nous étendrons pas dans le cadre de la présente Etude.

La stabilité de la circalation atmosphérique à la verticale $d$,un point est une règle générale à laquelle les exceptions sont relativement peu fréquentes.

Cette stabilité existe avec un degré de détail qui surprend:

-le vent peut rester constant d'une heure à l'autre, à une altitude donnée, souvent à moins de 100 mètres/minute près $(6 \mathrm{~km} / \mathrm{h}$. $)$;

-l'altitude à laquelle se produit une brusque variation de la force ou de la direction du vent demeure constante entre deux sondages souvent à moins de 200 mètres près.

Cette stabilité est:

-soit absolue: le vecteur à une altitude donnée: grandeur et direction-demeure sensiblement constant d'un sondage à l'autre;

-soit relative: l'évolution de la circulation se produit progressivement dans le même sens (variaton de la force et de la direction du vent): on retrouve des aspects caractéristiques des courbes $V(z)$ et $D(z)$ d'une courbe à l'autre.

En ce qui concerne les exceptions à cette règle nous ferons, en particulier, observer:

-que les remarques précédentes ne s'appliquent pas aux basses couches de l'atmosphère: jusqu'à $3-4 \mathrm{~km}$. d'altitude, qui pratiquement n'ont pas fait l'objet de mesures suivies de notre part.

Par ailleurs ce que l'on pourrait dénommer une turbulence à l'échelle synoptique(2) se manifeste dans certains cas qui semblent peu nombreux.

Nous donnerons ci-dessous quelques exemples caractéristiques de la stabilité de la circulation atmosphérique en altitude.

Nous présenterons tout d'abord trois exem-

(1) à quoi s'ajoutent environ 200 sondages effectués antérieurement dont une centaine en été.

(2) Se reporter à l'Etude sur: "Mesure précise du vent en altitude à l'aide de sondages rapprochés dans le temps "-mars 1956 - p. 50. ples comprenant à la fois les "courbes de travail" donnant la variation en fonction de l'altitude des trois éléments $D R$, $z D S$ et $V_{t}$ et les "courbes définitives" ces dernières donnant la variation en fonction de l'altitude des éléments classiques du vecteur vent: c'est-à-dire $V$ et $D$.

Nous donnerons ensuite, sans les commenter, 3 autres exemples.

$1^{\circ}-3$ sondages à haute altitude à 09.30 T.U. -12.50 TU. $-14.35 T U$. le 7 février 1957 (Figs. 2 et 3 ). $n^{\circ}$ 445-446-447.

Ces trois sondages recouvrent une période de 5 heures au cours de laquelle la circulation atmosphérique entre le sol et l'altitude $26-27 \mathrm{kn}$. est restée sensiblement constante.

Les vitesses du vent coincident à moins. de $100 \mathrm{~m} / \mathrm{minute}$ :

- pour les sondages $1(09.30)$ et $2(12.50)$ entre les niveaux 9 et $12,5 \mathrm{~km}$. puis 16,5 . et $23,5 \mathrm{~km}$.

- pour les sondages 2 et 3 entre les niveaux 3 et $7 \mathrm{~km}$. puis 12,5 et $16,5 \mathrm{~km}$., puis. 18 et $23,5 \mathrm{~km}$.

En ce qui concerne la direction du vent les courbes des sondages 2 et 3 sont en coïncidence, à moins de 10 degrés près, sur toute leur étendue, sauf au voisinage du niveau $17-18 \mathrm{~km}$. où la force du vent est minimum et inférieure à $500 \mathrm{~m} /$ minute $(30 \mathrm{~km} / \mathrm{h}$.).

La courbe de la direction du vent pour le sondage 3 est légèrement $\left(20^{\circ}\right.$ au plus) divergente des 2 courbes précédentes dans la troposphère.

En ce qui concerne les "courbes de travail " on peut faire les remarques suivantes.

Tout d'abord les courbes zDS sont pratiquement confondues à moins de $150 \mathrm{~m} /$ minute près, généralement jusqu'à l'altitude de $25 \mathrm{~km}$.

Les 3 courbes $D R(z)$ sont en bonne coincidence pratiquement jusqu'à la même altitude de $25 \mathrm{~km}$.

Il en est de même des 3 courbes de $V_{t}(z)$ jusqu'au niveau de $24 \mathrm{~km}$, sinon que la 


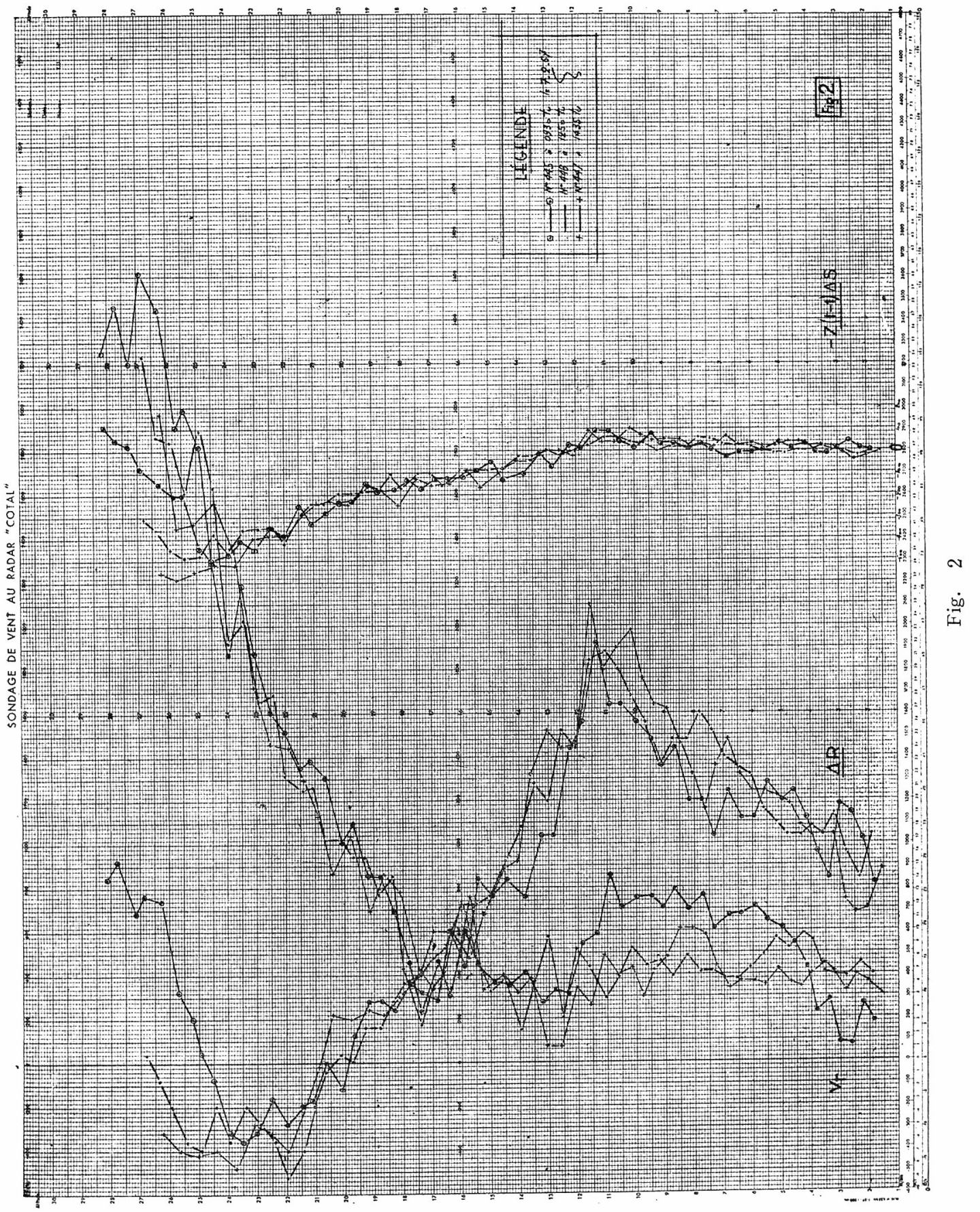


75 th Anniversary Volume of the Journal of the Meteorological Society of Japan

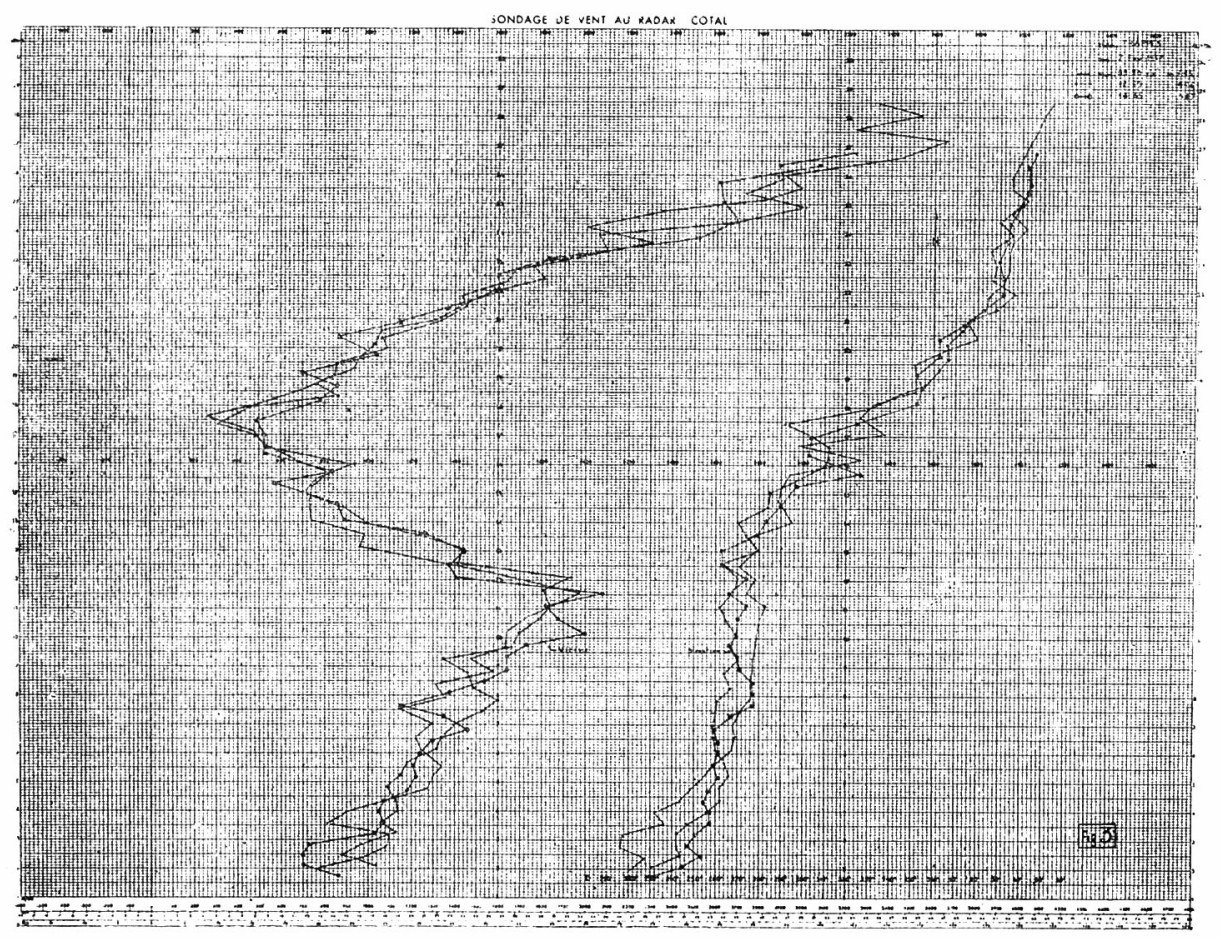

Fig. 3

courbe du sondage $1\left(n^{\circ} 445\right)$ donne un vent transversal plus fort entre les niveaux 4,5 et $11 \mathrm{~km}$. environ.

$2^{\circ}$-Evolution d'un courant " Jet " - dans la période de décroissance à la verticale de Trappes le 25 Janvier 1957 (Figs. 4 et 5).

Les courbes correspondant aux 7 sondages successifs ( $N^{\circ} 393$ à 399) exécutés le 25 Janvier 1957 entre 17.10 TU. et 01.46 TU. mettent en évidence ce que nous avons dénommé précédemment une évolution progressive-dans le même sens-de la circulation atmosphérique:

a) -Les courbes "définitives" mettent en évidance les caractères essentiels suivants du courant "Jet" dans sa période de décroissance:

-décroissance continue d'un sondage à l'autre de la force du vent au-dessous du niveau $11 / 11,5 \mathrm{~km}$, vent peu variable audessus de ce niveau;

- les courbes de la vitesse des différents sondages se raccordent très sensiblement, à une portion de droite peu inclinée entre les niveaux $11,2 \mathrm{~km}$ et $12 \mathrm{~km}$.

-la direction du vent est peu variable, avec toutefois-entre les niveaux $4 \mathrm{~km}$. et $11,5 \mathrm{~km}$. environ une rotation cyclonique progressive:-le vent s'écarte du Nord atteignant 20 à $25^{\circ}$ entre le premier et le dernier sondage.

b) -Sur les courbes de travail:

-la diminution progressive simultanée d'un sondage à l'autre:

$$
\begin{aligned}
& \text { - de } D R \\
& \text {-et de } V_{i}
\end{aligned}
$$

-l'existence d'une couche-entre 11 et 12 $\mathrm{km}$.- de diminution rapide de $D R$ et de $V_{t}$,

- enfin la confusion des différentes courbes zDS et la valeur constamment négligeable de ce terme correctif, sont en fait une représentation, différente, mais aussi claire, des caractères décelés sus les courbes définitives.

Cet exemple est caractéristique de la mise en évidence, sur les courbes correspondant 


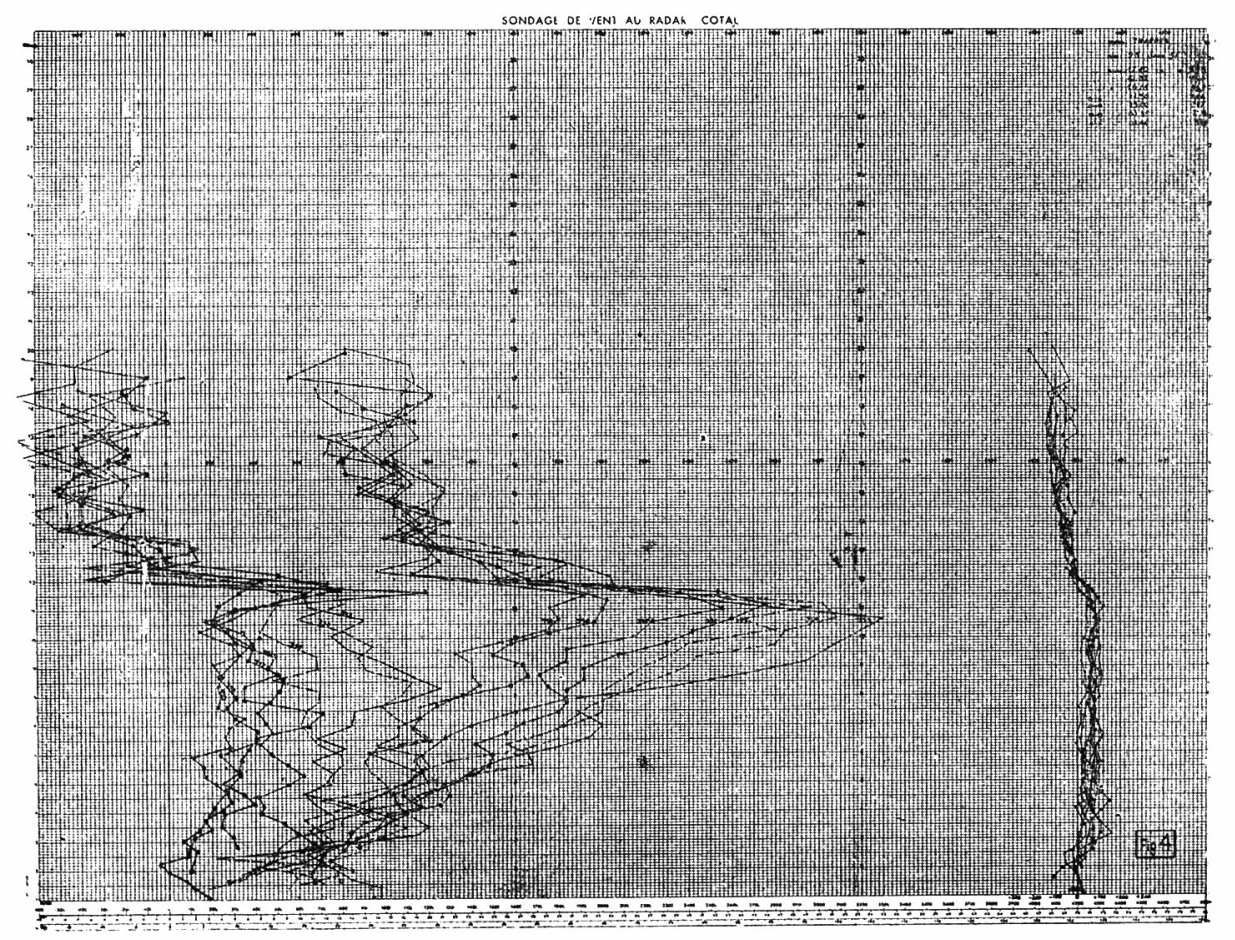

Fig. 4

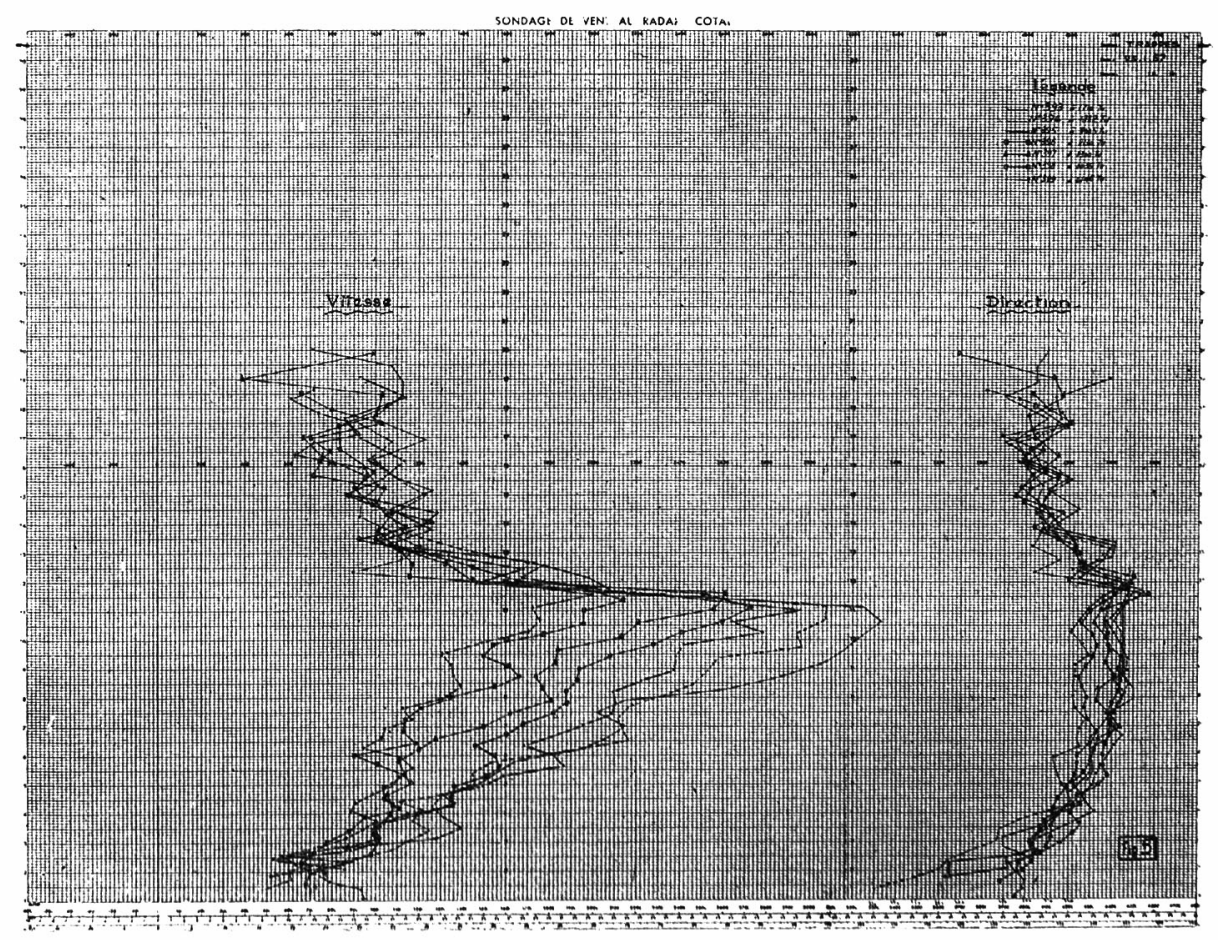

Fig. 5 
à des sondages successifs rapprochés dans le temps, de la précision pratique du radar lorsque la circulation atmosphérique est en évolution rapide.

Le caractère continu de cette évolution fait apparaître, par la faiblesse relative du décalage entre les courbes successives, en particulier aussi bien celles de $V(z)$ que celles de $D R(z)$, une précision pratique de l'ordre de $100 \mathrm{~m} /$ minute dans la mesure de la vitesse et de $10^{\circ}$ dans celle de la direction du vent.

$3^{\circ}-3$ sondages du 22 Janvier 1957. $\mathrm{N}^{\circ} 374$ à $12.35 T U .-375$ à $13,55 T U .-376$ à 16.00 TU. (Figs. 6 et 7 ).

Nous avons cru devoir présenter un exemple d'un cas particulier, à la vérité exceptionnel: c'est le seul que nous avons observé durant ces 6 mois où les 3 éléments du vecteur vent $D R, \mathrm{Vt}-z D S$ et particulièrement le dernier ont des valeurs du même ordre de grandeur et de plus sont fortement variables au voisinage d'une altitude particulère.

Le vent entre les niveaux $2 \mathrm{~km}$ et $5,5-$ $6,5 \mathrm{~km}$ décroît en force de $50 \mathrm{Km} /$ heure à $10 \mathrm{~km} /$ heure.

Sa direction de secteur SW jusque vers $4-5 \mathrm{~km}$ d'altitude passe brutalement à $E$ (par le Nord) entre ce niveau et l'altitude $6,5 \mathrm{~km}$.

Au-dessus du niveau $6,6 \mathrm{~km}$ la force du vent augmente rapidement jusqu'à atteindre $100 \mathrm{~km} /$ heure environ à l'altitude $8,5 \mathrm{~km}$. pour décroître jusqu'à une force de $12 \mathrm{~km}$ /heure environ au niveau $12 \mathrm{~km}$.

Au-dessus de $12 \mathrm{~km}$. d'altitude le vent croît lentement avec l'altitude jusqu'à la limite du sondage $(24-29 \mathrm{~km})$.

La direction du vent au-dessus du niveau $6,5 \mathrm{~km}$. vire vers le Nord:

-tout d'abord lentement: de $90^{\circ} \mathrm{E}$ au niveau $12,5 \mathrm{~km}$.

- puis rapidement au-dessus de ce dernier niveau pour devenir $W S W\left(230-250^{\circ}\right)$ à partir du niveau $14 \mathrm{~km}$.

La constance dans l'intervalle des mesures
-3 heures $1 / 2$ - de cette circulation cependant très différenciée avec l'altitude se marque par la coincidence des courbes $V(z)$ et $D(z)$ :

En ce qui concerne la vitesse du vent, cette coïncidence a lieu à moins de $100 \mathrm{~m} /$ minute près, sauf dans la couche $17-19 \mathrm{~km}$.

Les courbes de la direction du vent sont, de même, pratiquement confondues, sauf dans la couche 11,5-14 km à l'intérieur de laquelle la vitesse du vent est inférieure à $500 \mathrm{~m} /$ minute.

Il faut, en outre, noter le point qui est en faveur de la précision des mesures:

Le ballon est resté au voisinage du zénith du radar: sa projection horizontale s'écartant de moins de $7 \mathrm{~km}$, de celui-ci entre le sol et l'altitude de $23 \mathrm{~km}$. environ.

Il en est résulté entre 2 minutes successives de très fortes rotations du radar:

C'est ainsi que $D \alpha$ prend les valeurs suivantes:

Valeurs de $D \alpha$ en millièmes et en degrés

\begin{tabular}{c|c|c|c}
\hline $\begin{array}{c}\text { Alitude en } \\
\text { mètres }\end{array}$ & Sondage 374 & Songage 375 & Sondage 376 \\
\hline 7709 à 8067 & $236\left(13^{\circ}\right)$ & - & - \\
7817 à 8271 & - & $260\left(14^{\circ}\right)$ & - \\
7883 à 8365 & - & - & $318\left(18^{\circ}\right)$ \\
\hline 8067 à 8503 & $570\left(32^{\circ}\right)$ & - & - \\
8271 à 8689 & - & $640\left(36^{\circ}\right)$ & - \\
8365 à 8866 & - & - & $450\left(25^{\circ}\right)$ \\
\hline 8503 à 8896 & $780\left(43^{\circ}\right)$ & - & - \\
8689 à 9206 & - & $734\left(41^{\circ}\right)$ & - \\
8866 ¿̀ 9366 & - & - & $470\left(26^{\circ}\right)$ \\
\hline 8896 à 9315 & $396\left(22^{\circ}\right)$ & - & - \\
9206 à 9605 & - & $126\left(7^{\circ}\right)$ & - \\
9366 à 9877 & - & - & $330\left(19^{\circ}\right)$ \\
\hline
\end{tabular}

Soit un rotation totale en degrés, pour moins de 2000 mètres de dénivellation respectivement égale à: $110^{\circ}, 98^{\circ}$ et $90^{\circ}$ pour les sondages 374,375 et 376 .

Malgré ces fortes valeurs de $D \alpha$ et aussi les fortes valeurs de l'angle de site (environ $70^{\circ}$ ) et des varitions de celui-ci (jusqu'à $7^{\circ}$ en une minute) les valeurs calculées des 


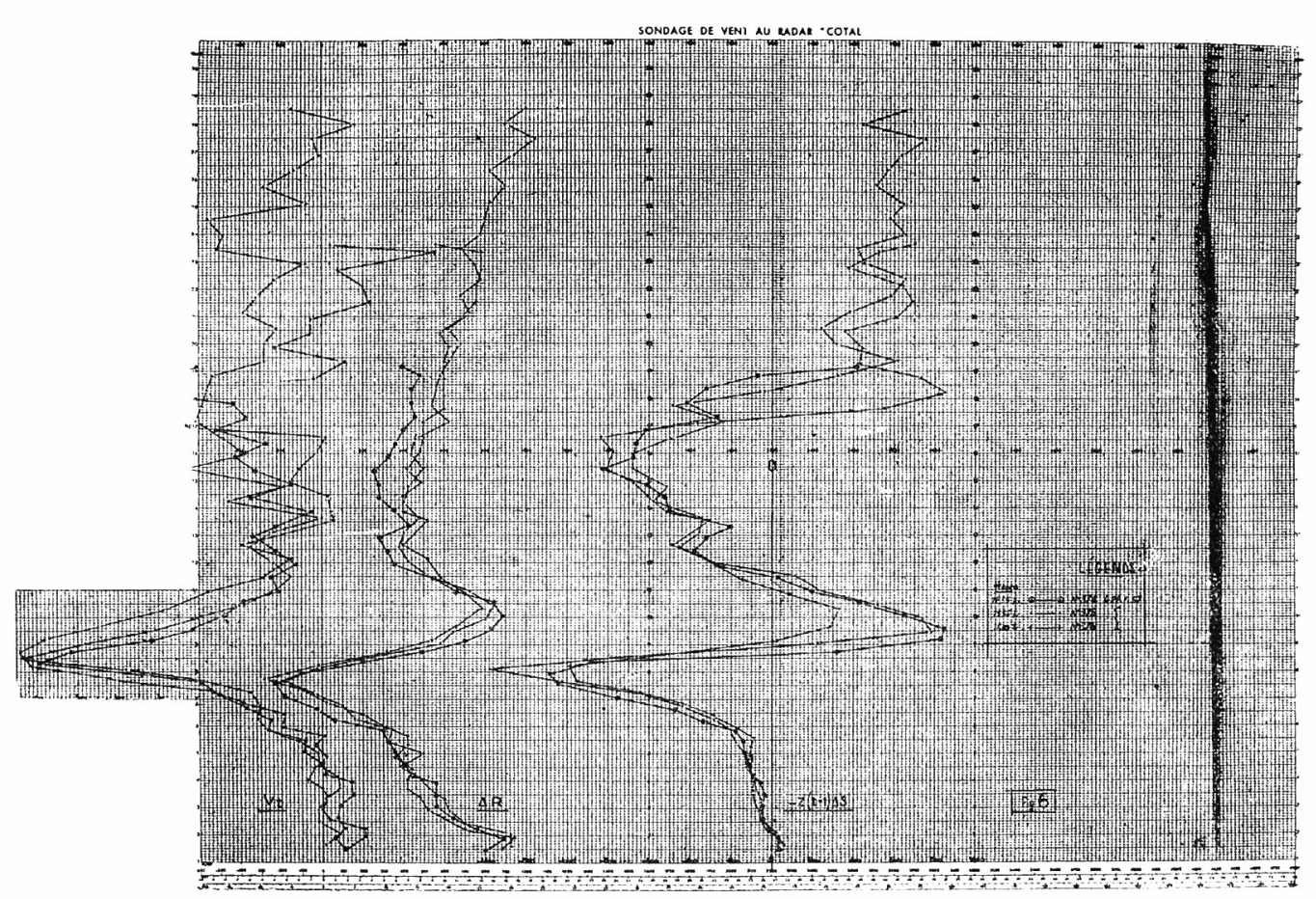

Fig. 6

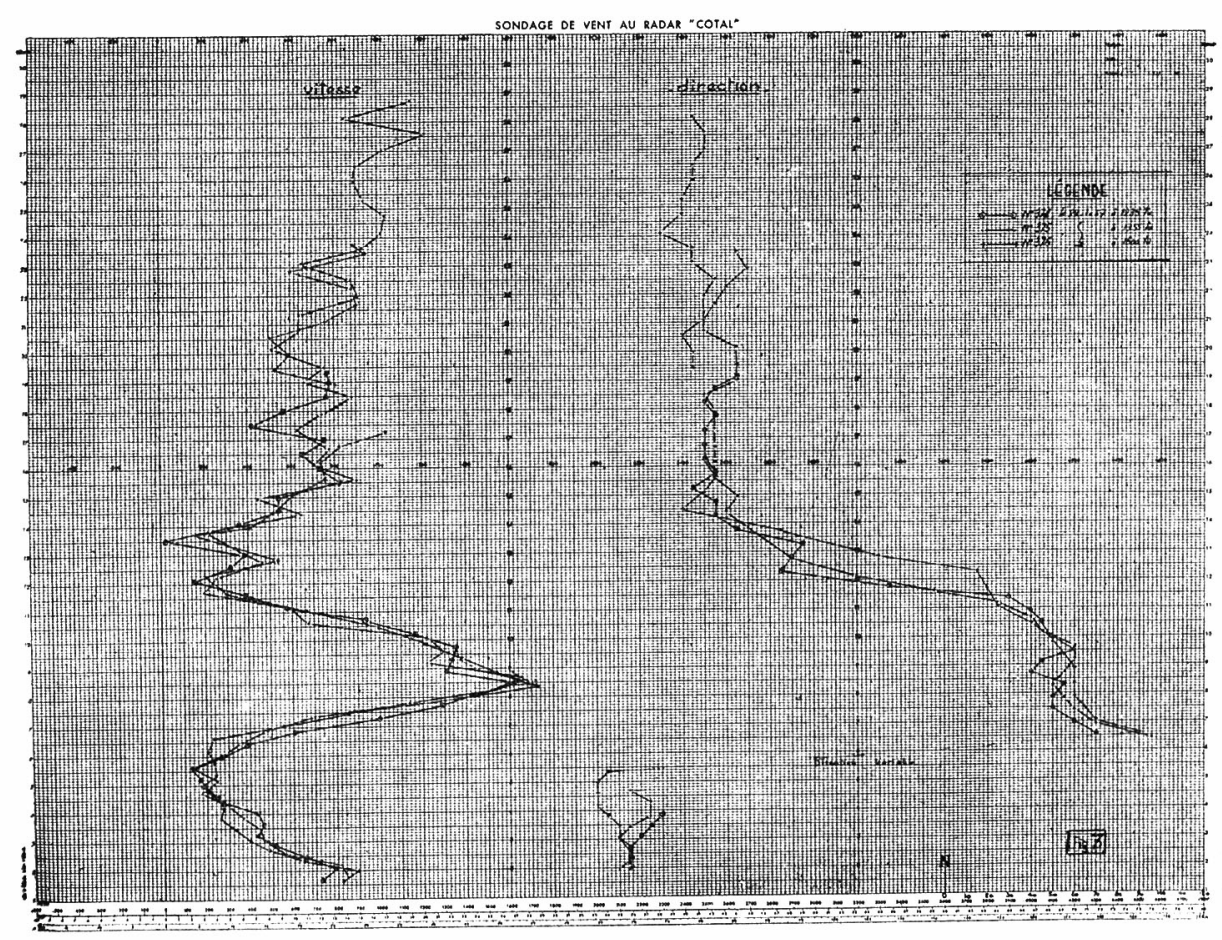

Fig. 7 
75 th Anniversary Volume of the Journal of the Meteorological Society of Japan

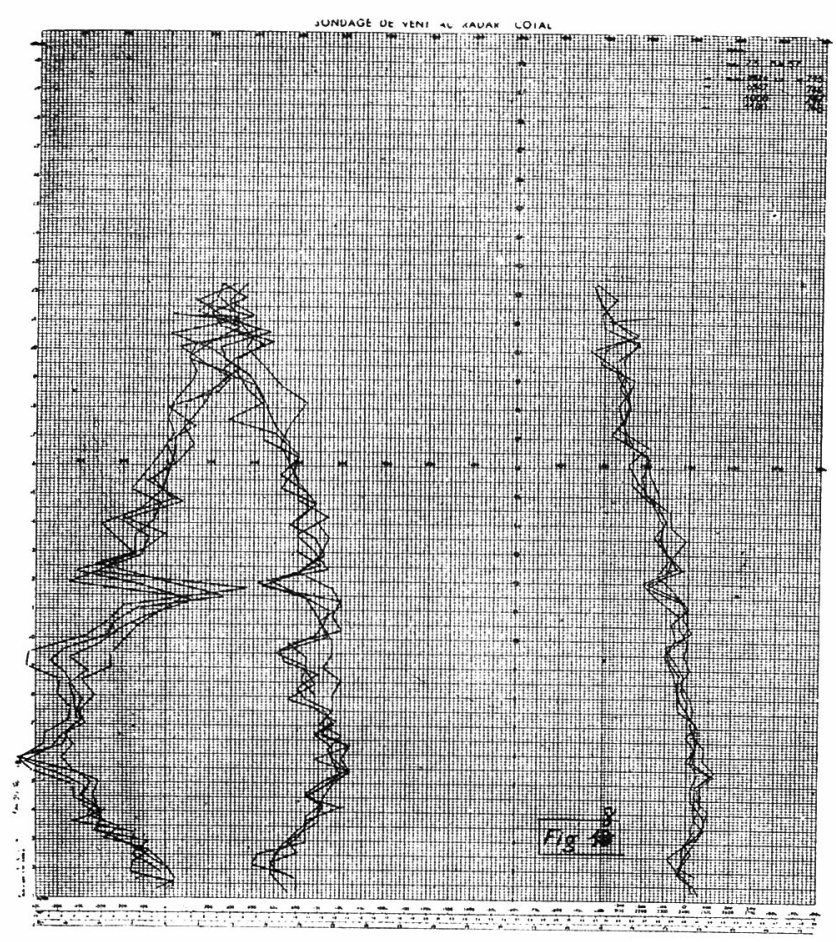

Fig. 8

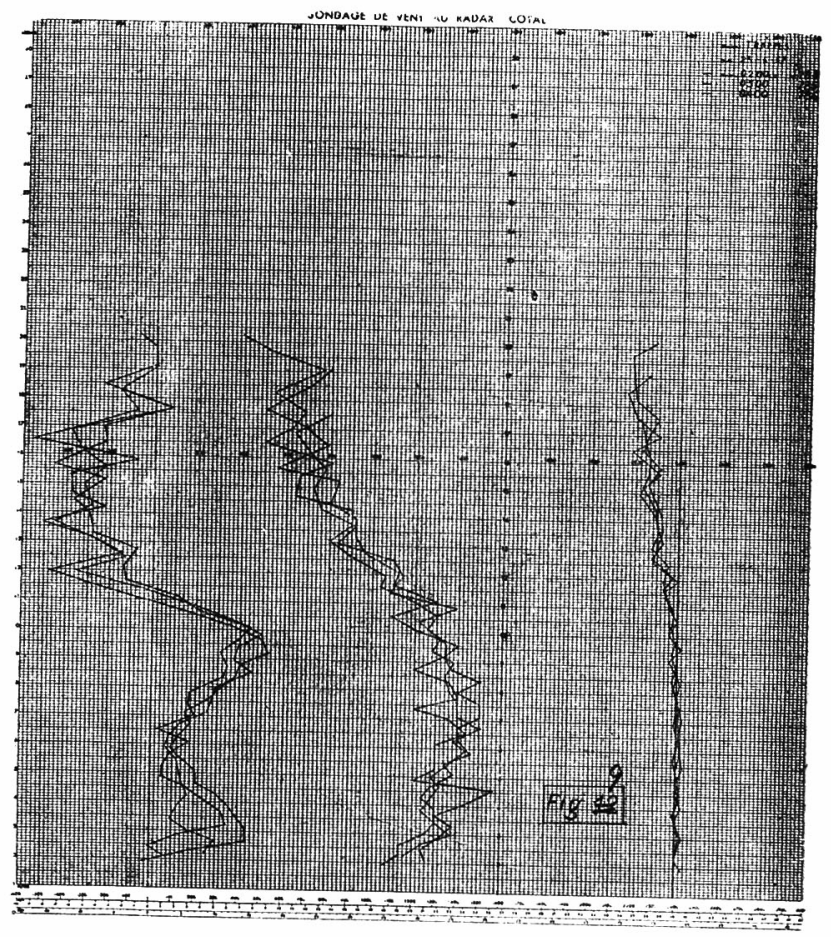

Fig. 9 éléments du vecteur vent-direction et vitesse-restent constantes.

L'examen des " courbes de travail" permet de prévoir les conclusions qui viennent d'être rapportées.

La constance d'un sondage à l'autre des éléments: $\mathrm{DR}, \mathrm{Vt}-z D S$ entraine la superposition-relativement excellente-des 3 courbes $D R(z), V t(z)$ et $-z D S(z)$.

On remarquera, en particulier, la constance d'un sondage à l'autre, de l'altitude des niveaux des extrêmes de ces courbes.

De plus la précision des mesures se marque entre les niveaux 8 et 12 $\mathrm{km}$ environ par un décalage cohérent des courbes des 3 sondages successifs l'une par rapport à l'autre.

Les 3 exemples ci-dessus établissent suffisamment; semble-t-il, le bien-fondé de notre hypothèse sur les "éléments suffisants " à définir le vecteur vent, de plus, ils permettent d'apprécier pratiquement le degré de précision des mesures.

$4^{\circ}-4$ sondages exécutés le $23 \mathrm{Mai}$ 1957 (Fig. 8-Courbes de "travail": $\left.V_{t},-D R,-z D S\right) \mathrm{n}^{\circ} 746$ à $0907 T U$ 747 à $1000 T U 748$ à $1100 T U$.

$5^{\circ}-3$ sondages exécutés le $25 \mathrm{Mai}$ 1957 (Fig. 9-Courbes de travail)n ${ }^{\circ}$ 784 à $0200 T U, 785$ à $0300 T U$. Ces 7 sondages font partie d'un ensemble de 45 sondages successifs exécutés en 48 heures: entre le 23 Mai 1957 à $0820 T U$ et le 25 Mai à $0700 \mathrm{TU}$.

$\mathrm{Au}$ cours de ces 2 journées la verticale de Trappes était voisine d'une dépression relativement étroite et mobile, il en résulte que la variabilité du vent a été en général assez importante, les deux exemples retenus correspondent à des périodes de stabilité de la 
Estimation pratique des erreurs commises dans la mesure du vent

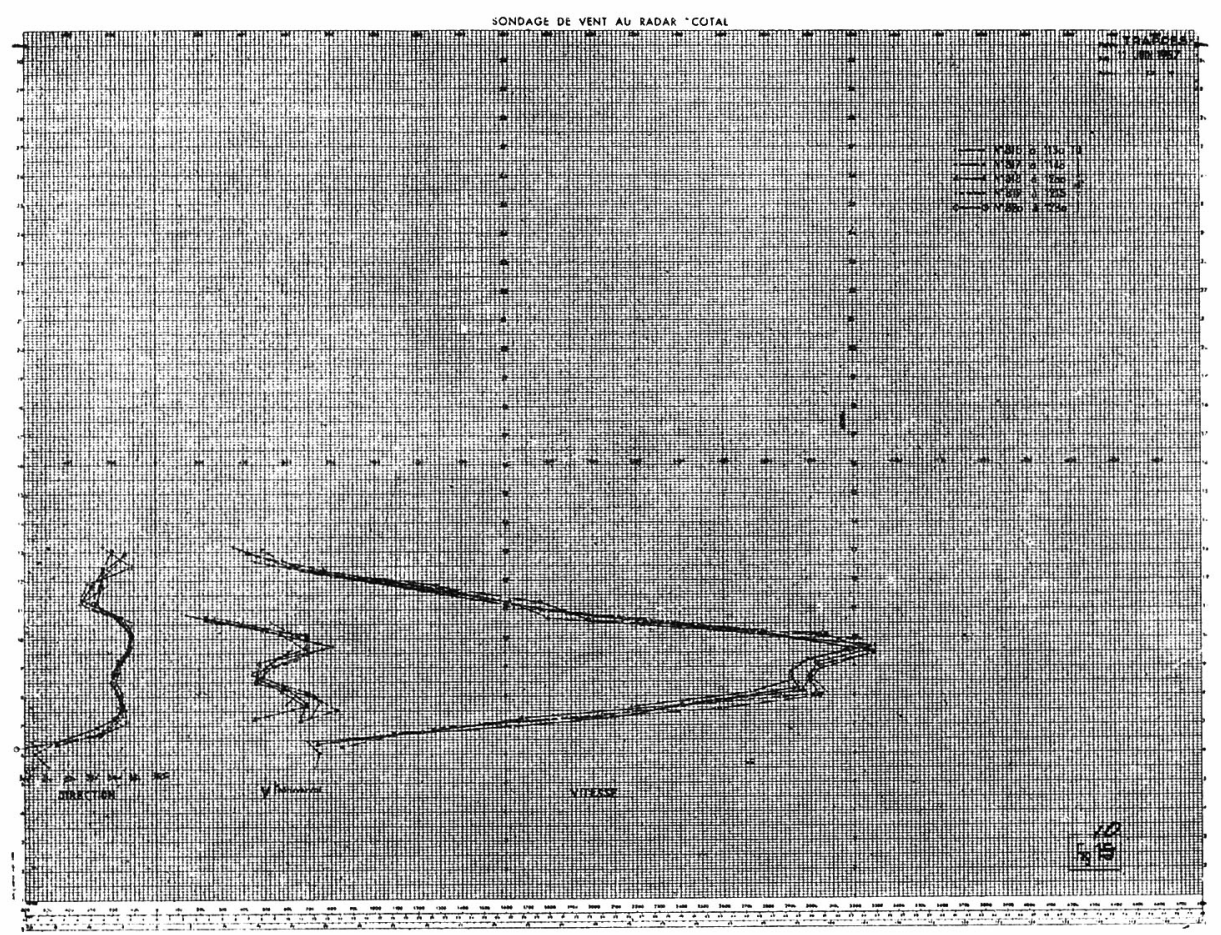

Fig. 10

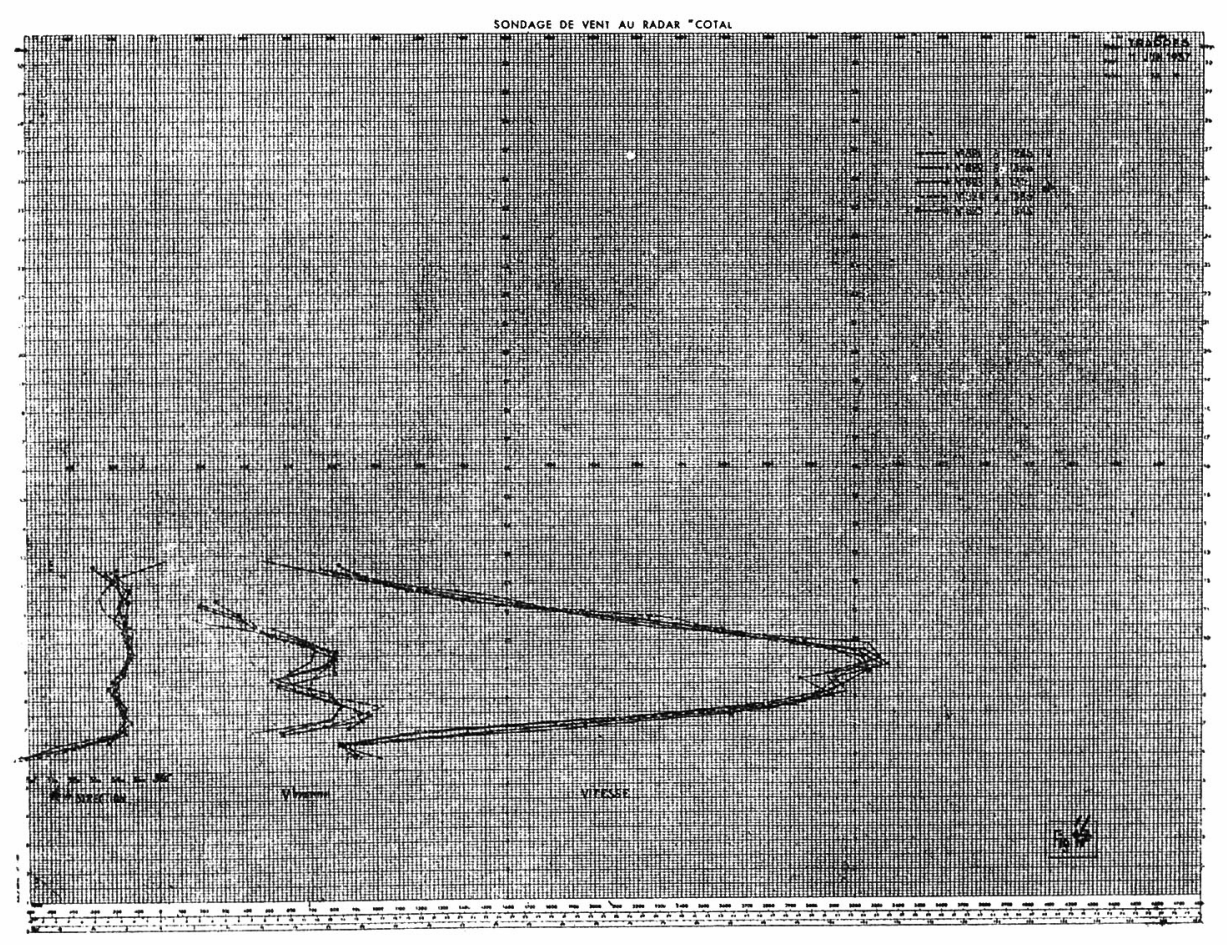

Fig. 11 
circulation atmosphérique.

$6^{\circ}-10$ sondages successifs exécutés de 15 en 15 minutes dans la partie centrale d'un courant jet entre les altitudes $6 \mathrm{Km}$ et $11 \mathrm{Km}$ environ. (Fig. 10 et 11)

Chacune de ces figures représente la variation en fonction de l'altitude: de la vitesse et de la direction du vecteur vent total d'une part, et de la grandeur du vent transversal d'autre part.

Appreciation de la précision des mesures par l'assimilation, suivant les cas, de la vitesse du vent soit à la grandeur $D R$, soit à la vittesse transversale

Nous reproduisons (Fig. 12) l'ensemble de courbes(1) "définitives" se rapportant au sondage $\mathrm{n}^{\circ} 445$ du 7 Février 1957 à 09.30 $T U$.

Ces courbes donnent, en fonction de l'altitude la variation des grandeurs $D R, V t$, et $D^{\circ}$,

Ce sondage a atteint l'altitude de $28,5 \mathrm{~km}$.
La circulation atmosphérique se caractérisait, ce jour là, par son caractère très différencié entre la troposphère et la "haute" atmosphère (entre 20 et $30 \mathrm{~km}$ d'altitude):

- vent de secteur $S W$ dans la troposphère correspondant à la phase finale d'un "courant Jet";

-vent s'établissant ensuite progressivement au secteur $N E$ en augmentant de force avec une rotation s'accentuant à partir de $16,5 \mathrm{~km}$ d'altitude: la vitesse du vent atteint son maximum: 3600 mètres/minute (216 km/heure) au sommet du sondage à l'altitude $27-29 \mathrm{~km}$.

On constate sur ce graphique:

-que la vitesse du vent est, avec une excellente approximation, mesurée par:

a) $D R$ jusqu'à l'altitude $17 \mathrm{~km}$.

b) par $V t$ au-dessus de l'altitude $17 \mathrm{~km}$.

Entre les altitudes 16,5 et $17,5 \mathrm{~km}$, le vent atteint sa force minima qui ne s'écarte, d'ailleurs pas sensiblement, en valeur absolue de $D R$ ou de $V_{t}$.

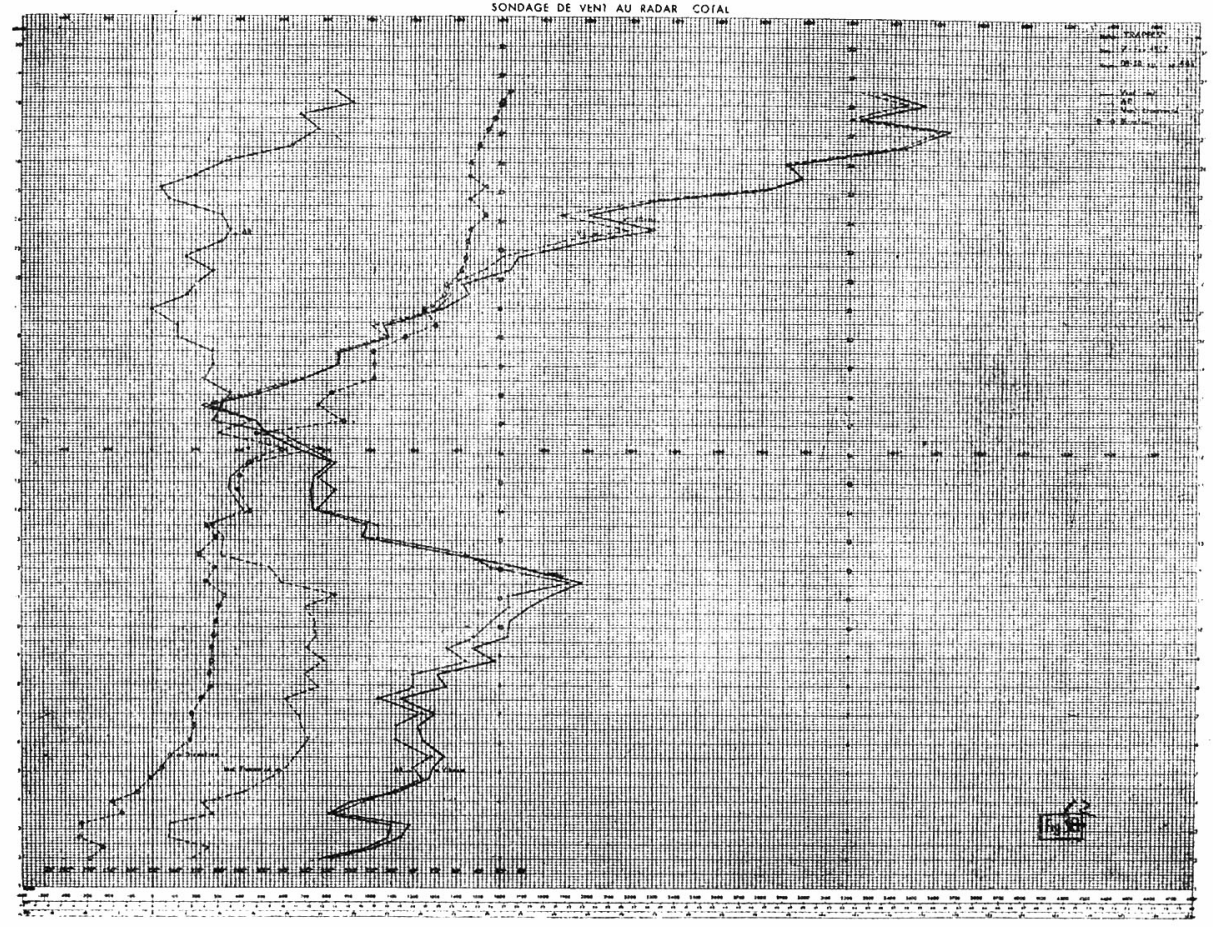

Fig. 12

(1) sauf la courbe.du vent raidal $V r$ trop peu distincte de celle de $D R$ jusqu'au niveau $16 \mathrm{~km}$ environ. 
Cet excellent "ajustement" de la courbe $V(z)$ d'abord par celle de $D R(z)$ puis celle de $V_{t}(z)$ est lié à ce type de circulation.

Il peut se justifier ainsi:

-la projection horizontale de la trajectoire du ballon (Fig. 13) est constituée par 2 éléments de courbes à faible courbure et d'orientation très différente séparés par une faible longueur de courbe à forte courbure.

On peut établir facilement ${ }^{(1)}$ que si l'angle (1) du rayon vecteur $R b$ avec la tangente à la trajectoire:

- est inférieur à $24^{\circ}$ l'erreur commise en assimilant $V$ à $V r$ est inférieur à $10 \%$ de $V$.

De plus, lorsque le vent est généralement croissant avec l'altitude comme c'est le cas de l'exemple précité, la grandeur $D R$ est sensiblement une aussi bonne approximation de la valeur du vent que $V r^{(2)}$.

a) lorsque l'angle $\omega$ est supérieur à $64^{\circ}$ c'est alors $V_{t}$ qui joue le rôle de $D R$ (de $V_{r}$ ).

b) pour $24^{\circ}<\omega<66^{\circ}$

$V_{t}$ et $V_{r}$ interviennent par des effets du même ordre dans la valeur de $V$.

c) enfin si le sondage était poursuivi et si la circulation stratosphérique supérieure ne changeait pas sensiblement d'orientation, il est clair que l'angle $\omega$ diminuant progressivement atteindrait à nouveau la valeur $24^{\circ}$ au-delà de laquelle c'est à nouveau $V_{r}$. (et $D R$ ) qui "ajusterait" la force du vent. ${ }^{(3)}$

\section{Remarque :}

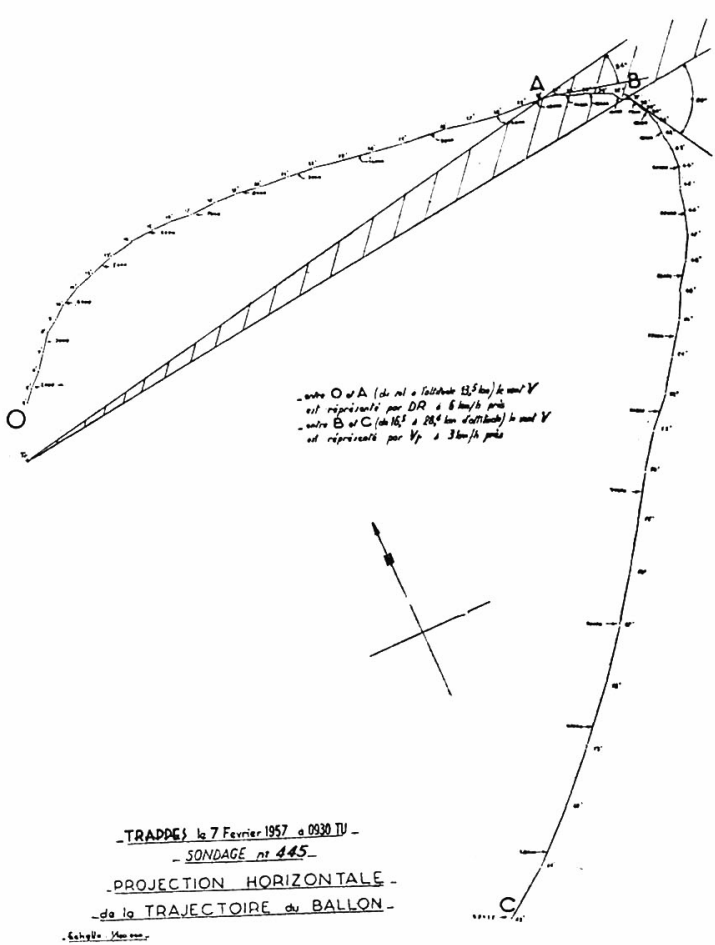

Fig. 13

(1) voir plus haut pages 3 et 4 .

(2) $V r=D R \cdot \cos . S-z D S$

Ce qui peu s’écrire : $V r=D R-D R(1-\cos . S)-z D S$ Les deux terme "correctifs" $D R$ (1-cos.S) et $z D S$ sont de signe contraire. Voir l'exemple du sondage $\mathrm{N}^{\circ} 426$ (Fig. 14)

(3) Cette remarque est susceptible, semble-t-i1 de recevoir des applications pratiques en exploitation courante:

Il est en effet possible, à l'aide des documents synoptiques disponibles dans les stations d'observation du réseau et relatifs au réseau précédent-essentiellement les cartes Contours 700-500-300 et 200 millibars-de prédéteminer:

Les niveaux entre lesquels on pourra utiliser soit $D R$, soit $V_{t}$ comme mesure approchée du vent.

Si la circulation atmosphérique est en période d'évolution rapide on peut tenir compte de cette évolution déterminée, par exemple, à partir du déplacement des centres d’action ou des surfaces frontales.

Il n'mporte, en effet, de disposer en ce qui concerne la vitesse du vent que d'une prévision assez grossière, plutôt une évolution qualitative. Il en est de même en ce qui concerne la direction du vent. 


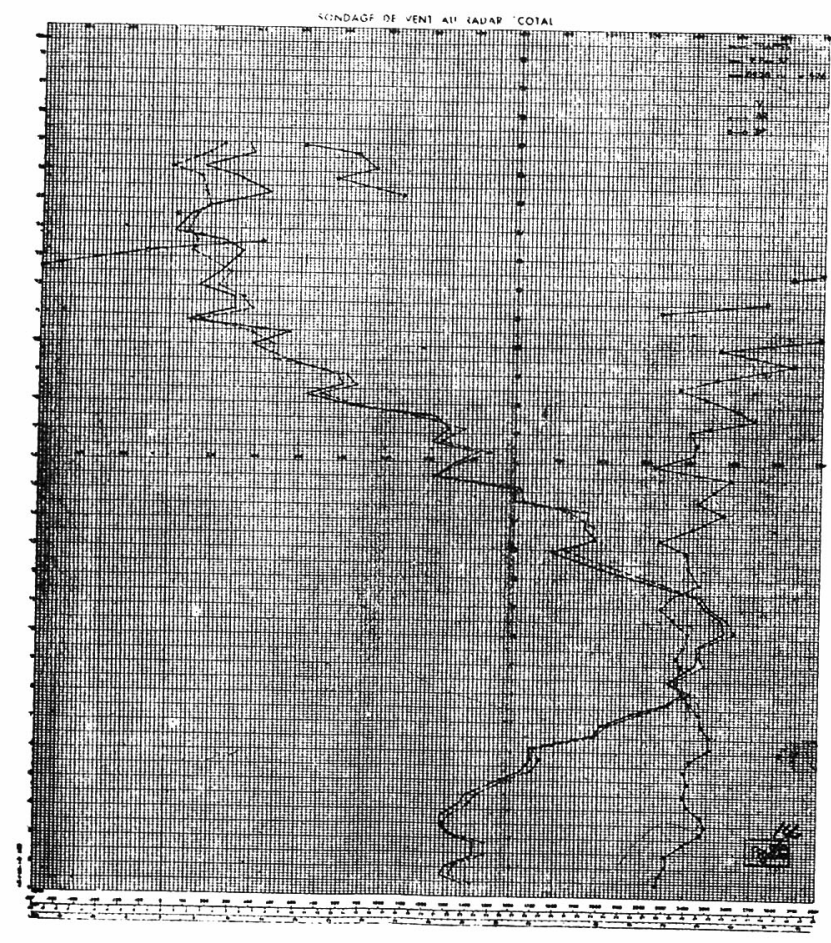

Fig. 14

\section{Conclusion :}

Application pratique a la détermination de la prècision instrumentale du radar.

On peut faire les remarques suivantes:

$1^{\circ}$-Lorsque la grandeur du vent est ajustée par $D R$, la précision mise en évidence par la comparaison des courbes correspondant à des sondages rapprochés dans le temps mesure la précision totale de la télémétrie du radar: télémétrié électronique jeux des mécanismes, procédé de lecture du radar, erreurs dues à l'agitation du réflecteur.

Par exemple, si cette précision est de 100 $\mathrm{m} /$ minute, on peut en conclure que précision totale de la télémétrie du radar est de 50 métres.

$2^{\circ}$-Lorsque la grandeur du vent est ajustée par le vent transversal $V_{t}=R$. $\times$ $\cos S D \alpha$ il est facile de voir que l'on peut calculer une borne surpérieure pratique (90\% des mesures inférieures à cette borne par exemple) de l'erreur totale commise dans la mesure de l'angle d'azimut: soit $\delta(V)=200 \mathrm{~m} /$ minute

la précision mise en évidence par la comparaison des courbes dans la mesure de la vitesse du vent.

On a par hypothèse

$$
\delta(V) \# \delta\left(V_{t}\right)
$$

or

$$
\delta\left(V_{t}\right) \# 2 R . \cos . S . \delta(\alpha)
$$

$\delta(\alpha)$ désignant l'erreur commise dans la mesure de l'angle d'azimut $\alpha$.

Si l'on exprime $\delta(\alpha)$ en $1 / 1000$ de radian et $R$. cos. $S$ en $\mathrm{km}$

$$
\delta \alpha=\frac{\delta\left(V_{t}\right)}{2 \cdot R \cdot \cos . S}
$$

par exemple: avec $R$. cos. $S=50 \mathrm{~km}$.

$$
\delta \alpha=\frac{200}{100}=\frac{2}{1000} \text { de radian }
$$

C'est là-sensiblement-la borne supérieure de l'erreur maximum totale mise en évidence pour les mesures/en ce qui concerne le radar utilisé de l'angle d'azimut.

\section{Appendice}

\section{Remarque:}

Signification de la vitesee transversle $V_{t}$ et interprétation des points caractéristiques de la courbe $V_{t}=f(z)$

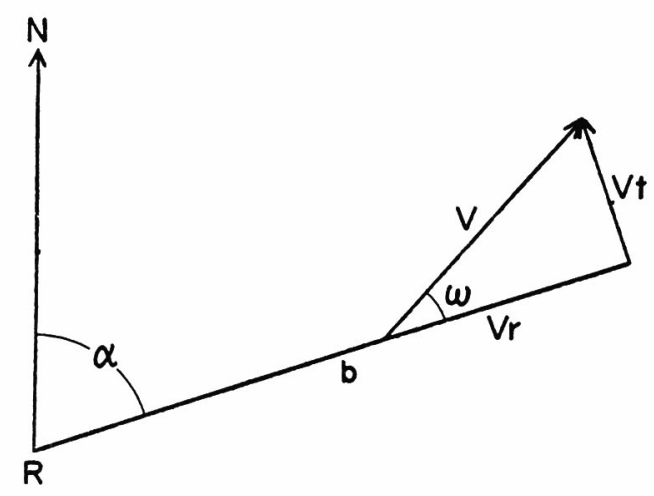

Fig. $\quad 15$

Le vent tranversal $V_{t}$ mesure la tendance à la rotation avec l'altitude des lignes de flux du vent qui sont elles-mêmes confondues avec les isobares ou,les lignes de niveau des surfaces isobariques en régime permanent seule

varia

tance

Les

minin

les $n$

chang

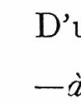

et un

circu

$-e$

tude

Les

$V_{t}=$

de tr

culat

L'e

$\mathrm{du}$

versa

dans

courl

cet é

(1)

versa 
seulement.

On rappelle (Fig. 21 ci-dessus) / que la direction du vent $\mathrm{D}^{\circ}$ est donnée par une des 2 formules:

$$
\begin{gathered}
D=\alpha \pm \omega \quad \text { ou } \quad D=\alpha \pm \omega+\pi \\
\text { avec } \operatorname{tg} \omega=\frac{V_{t}}{V_{r}}
\end{gathered}
$$

L'expérience montre que dans la grande majorité des cas et ceci d'autant plus que l'altitude augmente ${ }^{(1)}$ la rotation du radar entre 2 minutes successives (la valeur de $D \alpha)$ est inférieure à un degré et que par contre la variation de $\omega$ dépasse plusieurs degrés.

Il en résulte pratiquement que le sens de rotation absolue du vent entre deux minutes successives est lié au signe de la variation de $\operatorname{tg} \omega$ et le plus souvent au signe de la variation de $V_{t}$.

D'où cette conclusion de grande importance pratique:

Les altitudes des extremum (maximum et minimum) de la courbe $V t=f(z)$ définissent les niveaux où la circulation atmosphérique change de courbure.

D'une façon plus précise:

- $\dot{a}$ altitude croissante: entre un minimum et un maximum de la courbe $V_{t}=f(z)$ la circulation est anticyclonique.

-elle est cyclonique quand on passe $\grave{a}$ altitude croissante d'un maximum à un minimum,

Les altitudes des extremum de la courbe $V_{t}=f(z)$ correspondent à celles des couches de transition entre les deux types de circulation.

\section{L'expérience montre que:}

-le passage d'un régime anticyclonique du vent à un régime cyclonique ou vice versa est souvent très brutal et se produit dans des couches peu épaisses: les différentes courbes présentées sont caractéristiques à cet égard: le changement de régime s'effec-

(1) pour une même grandeur $V_{t}=A$ du vent transversal la grandeur de $D \alpha$ varie comme $\frac{1}{R}$. tue généralement d'une minute à l'autre(2). De plus, en suivant de seconde en seconde, sur le cadran donnant la valeur de l'azimut le déroulement d'un sondage au voisinage d'un tel niveau de transition (repéré grâce aux sondages antérieurs) nous avons été frappés par la rapidité-moins de 10 secondes-avec laquelle la rotation du cadran ayant changé de sens, la vitesse de rotation s'établit à nouveau à une valeur appréciable.

De plus, il y a généralement conservation (ou variation lente progressive) d'un sondage à l'autre pendant plusieurs heures de l'altitude de ces niveaux de transition.

Ces remarques appliquées à l'examen différentes courbes présentées mettent en évidence:

-la structure feuilletée fine (intéressant des couches ayant parfois moins de $1 \mathrm{~km}$. d'épaisseur) de l'atmosphère.

Entre 2 couches le vecteur vent varie, non seulement en grandeur mais aussi en direction (c'est là le "shear" selon la terminologie anglaise);

-la permanence ou l'évolution progressive de cette structure feuilletée fine pendant un intervalle dépassant plusieurs heures.

En d'autres termes:

-même des "détails" de la circulation atmosphérique en altitude ont un caractère synoptique qui se retrouve d'un réseau d'observation à l'autre;

-la “turbulence atmosphérique" n'intervient généralement(3) qu'à une échelle de temps et d'espace (en altitude et dans un

(1) on passe d'une minute à l'autre à une valeur de $\alpha$ positive, par exemple, de plusieurs millièmes à une valeur négative du même ordre de grandeur,

(2) Il y a quelques exceptions, se reporter à l’Etude sur : "Mesure précise du vent en altitude à l'aide de sondages rapprochés dans le temps", Mars 1956 p. 9. B. 2, p. 12. II. 4-2, p. 13. 
75 th Anniversary Volume of the Journal of the Meteorological Society of Japan

plan horizontal) inférieure à l'échelle des mesures actuelles: couches de 300 à 500 mètres d'épaisseurs, vitesse du vent appréciée à $6 \mathrm{~km} /$ heure près ${ }^{(1)}$

(1) Ceci confirme, semble-t-il, le bien-fondée de 1'hypothèse sur un "arrangement privilégié des hov vitesses conduisant à un minimum d’énergie dissipé par turbulence". Cette hypothèse est à la base d'une théorie sur la circulation générale de l'atmosphère élaborée par 2 météorologistes français MM. WERHLE et DEDEBANT.

Voir en particulier “Cours de Météorologie à l’Ecole Supérieure d’Aéronautique-1938” par Ph. WERHLE. 